\title{
Detection of Septata intestinalis in Stool Specimens and Coprodiagnostic Monitoring of Successful Treatment with Albendazole
}

\author{
Rainer Weber, Bärbel Sauer, Max A. Spycher, \\ Peter Deplazes, Ruth Keller, Rudolf Ammann, \\ Jakob Briner, and Ruedi Lüthy
}

From the Departments of Medicine (Divisions of Infectious Diseases and
Gastroenterology) and Pathology. University Hospital, Zurich: and the
Institute of Parasitology and Laboratory of Electron Microscopy.
University of Zurich, Zurich, Switzerland

\begin{abstract}
We describe two patients with AIDS and chronic diarrhea in whom the microsporidian Septata intestinalis was detected with use of light and electron microscopic coprodiagnostic techniques. The ultrastructure of the microsporidian spores found in their stool specimens was distinctly different from that of Enterocytozoon bieneusi, another intestinal microsporidian found in patients infected with human immunodeficiency virus. Electron microscopic examination of duodenal biopsy specimens available from one of the patients enabled identification of $S$. intestinalis and confirmed the similarity of spores found in feces and in duodenal tissue. Both patients' diarrhea stopped when they were treated with albendazole. Coprodiagnostic monitoring indicated disappearance of the parasites and allowed the diagnosis of a relapse in one patient, who responded well to a second course of treatment.
\end{abstract}

The use of novel histologic and coprodiagnostic techniques has improved the diagnostic yield for human immunodeficiency virus (HIV)-infected patients with diarrhea; in particular, it has facilitated the identification of intestinal protozoa such as the microsporidia Enterocytozoon bieneusi and Septata intestinalis, which had been missed with use of routine examination methods [1-4]. Preliminary observations have indicated that treatment of septata infection with albendazole may be curative [5]. Therefore, early identification of this parasite could be beneficial for patients. We describe two patients with HIV-associated chronic diarrhea in whom $S$. intestinalis was identified by means of coprodiagnostic techniques. They were treated with albendazole and became asymptomatic. Coprodiagnostic monitoring indicated disappearance of the parasites and allowed detection of a relapse in one patient, who responded well to a second course of treatment.

A 27-year-old HIV-infected homosexual Swiss man was well until February 1992, when he began to have five to eight watery stools per day. Repeated stool examinations for ova and parasites (including cryptosporidia), a bacterial culture, and a Clostridium difficile toxin assay were negative. His $\mathrm{CD}^{+}$cell count was $0.05 \times 10^{9} / \mathrm{L}$. Antiretroviral therapy and prophylaxis for Pneumocystis carinii pneumonia were started. Diarrhea persisted and led to a weight loss of $10 \mathrm{~kg}$ by May 1993, when his weight was $48 \mathrm{~kg}$. Examination of

Received 29 October 1993; revised 24 January 1994

Grant support: This work was supported in part by the Swiss National Program for AIDS Research (grant no. 91-7066).

Reprints or correspondence: Dr. Rainer Weber, Division of Infectious Diseases, Department of Medicine, University Hospital. CH-809l Zurich. Switzerland.

Clinical Infectious Diseases 1994;19:342-5

(c) 1994 by The University of Chicago. All rights reserved 1058-4838/94/1902-0031\$02.00 stool samples then revealed cysts of Entamoeha coli and Entamoeba histolytica. No amebic cysts were detected after treatment with metronidazole $(500 \mathrm{mg}$ three times daily for 10 days), but diarrhea persisted. At that time, light microscopic examination of chromotrope-stained stool specimens [1] (previously not performed) revealed microsporidian spores measuring 1.2 to $1.5 \mu \mathrm{m} \times 2.5$ to $3.0 \mu \mathrm{m}$ (figure 1 ). No parasites were detected in urine specimens. In addition, adenovirus type 9 was cultured (with MRC-5 cells) from stool specimens and was identified by seroneutralization with use of polyclonal type-specific antibodies (American Type Culture Collection, Rockville, MD).

Electron microscopic examination of stool specimens confirmed that the microsporidian spore ultrastructure was characteristic of $S$. intestinalis [2-4]. The spores measured $0.97 \times$ $1.33 \mu \mathrm{m}$, and their polar tubules were arranged in one row (figure 2). We found differences between the spore sizes assessed by light microscopic and electron microscopic examination techniques, possibly because of the different fixation procedures. Specimens were prepared as follows. Large fecal particles in the stool suspension were removed by gravity sedimentation performed for 2 minutes, and the resulting supernatant was centrifuged at $5,500 \mathrm{~g}$ for 3 minutes. The pellet was fixed for 30 minutes at $4{ }^{\circ} \mathrm{C}$ with $3 \%$ glutaraldehyde in phosphate buffered saline and was postfixed for 30 minutes at $4^{\circ} \mathrm{C}$ with $2 \%$ osmium tetroxide in $0.1 \mathrm{M}$ cacodylate buffer $(\mathrm{pH}, 7.2)$. After overnight incubation in a $2 \%$ aqueous solution of uranyl acetate, the material was dehydrated with dimethoxypropane and embedded in an epoxy resin mixture (Epon 812: Fluka Chemie, Buchs, Switzerland). The sections were stained with lead citrate and examined with a transmission electron microscope (Philips EM400 HM; Philips, Eindhoven, the Netherlands).

In June 1993 the patient was treated with albendazole ( $400 \mathrm{mg}$ twice daily for 2 weeks); the diarrhea stopped within 


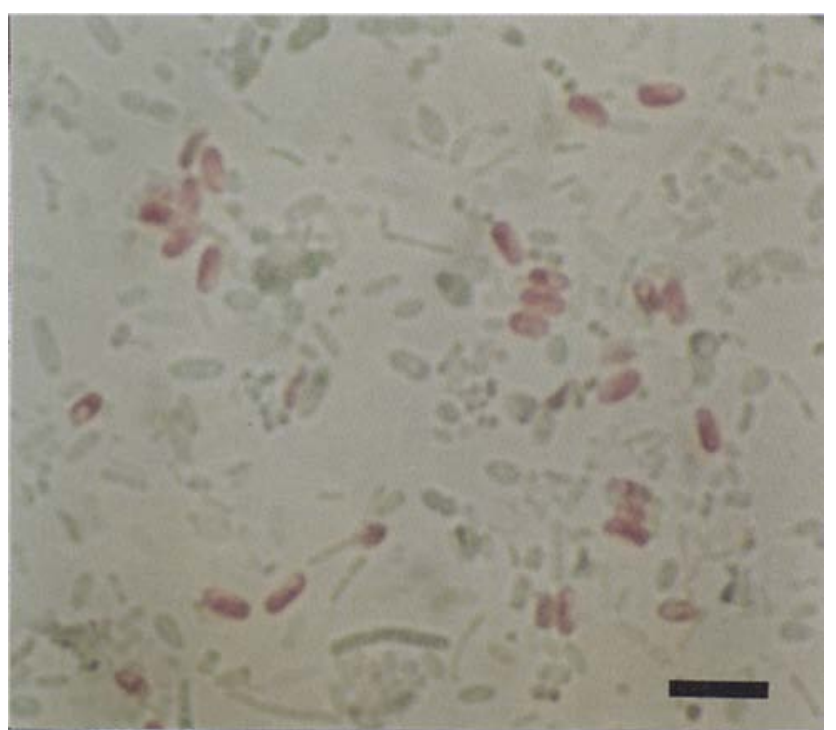

Figure 1. Smear of unconcentrated. formalin-fixed stool showed pinkish-red-stained S. intestinalis spores on light microscopic examination (chromotrope-based stain; oil immersion: bar $=5 \mu \mathrm{m}$ ).

5 days, and stool examination findings became negative. Disseminated atypical mycobacteriosis and cytomegalovirus chorioretinitis were diagnosed in September 1993, and treatments were initiated. In January 1994 he had no complaints. his weight was $57 \mathrm{~kg}$. and results of repeated stool examinations remained negative.

A 37-year-old HIV-infected heterosexual Swiss man had a history of $P$. carinii pneumonia, cerebral toxoplasmosis, and Kaposi's sarcoma. His $\mathrm{CD}^{+}$cell count was $0.02 \times 10^{9} / \mathrm{L}$. He received antiretroviral therapy as well as maintenance therapy for $P$. carinii pneumonia and toxoplasmosis. In May 1993 the patient experienced watery diarrhea, weight loss. nausea. and vomiting. His weight was $50 \mathrm{~kg}$. Microsporidian spores were found by light microscopic examination of chromotrope-stained stool specimens, and the microsporidian spore ultrastructure was confirmed by electron microscopic examination of stool specimens. An examination of urine specimens showed no parasites. Histologic examination of duodenal tissue sections obtained by gastroduodenoscopy (stained with hematoxylin-eosin and Brown-Brenn stain) showed mild inflammation (including slight intraepithelial infiltration by lymphocytes), villous atrophy. goblet cell depletion. and clusters of microsporidian spores within enterocytes and within macrophages of the lamina propria mucosae $[2,3]$. Microsporidia and small numbers of Giardia lamblia organisms were also detected in the duodenal aspirate. $S$. intestinalis was identified by electron microscopic examination of duodenal biopsy specimens [2-4] (figure 3).

Treatment with albendazole ( $400 \mathrm{mg}$ twice daily) was initiated, and diarrhea ceased. One week later, however, the patient was hospitalized because of sepsis syndrome. Fmpiric
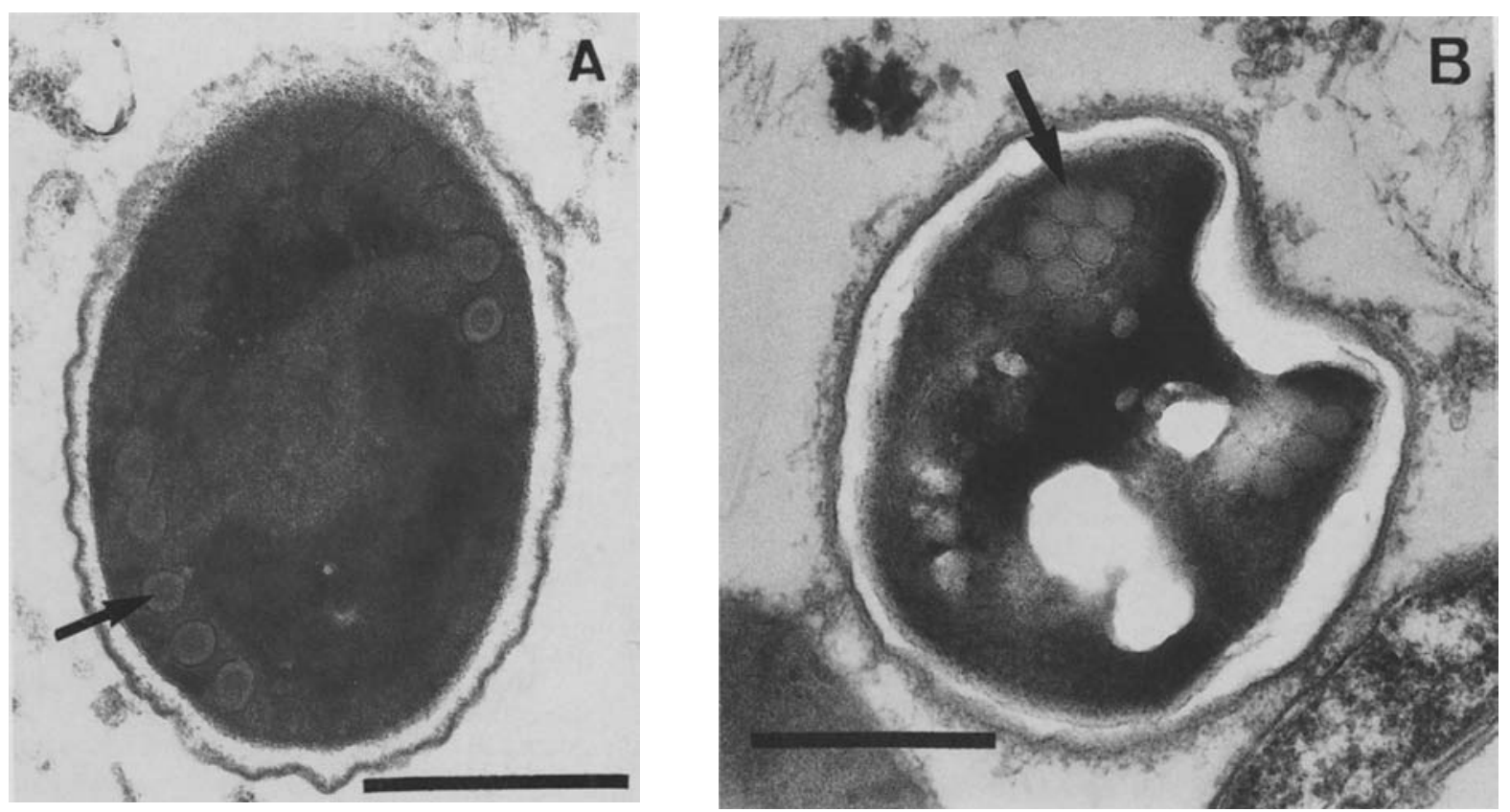

Figure 2. Electron micrograph of a stool specimen showing a spore of S. intestinalis ( $A$ ): a spore of $E$. biencusi ( $B$, reproduced from [9]) is shown for contrast. The ultrastructural characteristics are the polar tubules (arrow) coiled within the spore and a spore wall consisting of the plasma membrane, an electron-lucent endospore layer. and a dense outer spore coat. The arrangement of the polar tubules differs between $S$. intestinalis (tubules in one row [A. arrow]) and E. bieneusi (tubules in two rows [B, amow]). Micrograph of E. bienensi from reference [9]. Bar $=0.5 \mu \mathrm{m}$. 
intravenous antibiotic treatment (with imipenem) was started. Three blood cultures became positive for Staphylococcus aureus. Initially, the patient's condition remained critical, and he developed dyspnea and diffuse interstitial infiltrates that were evident on a roentgenogram of the chest. Cultures and a parasitological examination of bronchoalveolar lavage fluid (including a microsporidia-detection procedure $[6,7])$ were negative. Although albendazole was withdrawn at the time of hospitalization (after a I-week course), the microsporidian spores in stool samples disappeared during hospitalization. After a 3-week course of therapy with imipenem, the patient was discharged; he had no pulmonary or gastrointestinal symptoms. In July 1993 the patient again experienced nausea, vomiting, and diarrhea, and examination of stool samples again revealed $S$. intestinalis spores. A 2-week course of albendazole ( $400 \mathrm{mg}$ twice daily) led to clinical improvement, and stool samples became negative. During a 4-month follow-up period, the patient had no complaints and gained $21 \mathrm{~kg}$.

The identification of microsporidia depended on electron microscopic examination of biopsy specimens obtained by invasive procedures. However, initial detection of the parasite in tissue sections via light microscopy and in more easily obtainable bodily fluids (including stool specimens, duodenal aspirates, urine, bronchoalveolar lavage fluid, and conjunctival smears) via cytological examination is now readily accomplished $[1,6-9]$. Findings of light microscopic examination may even suggest a distinct microspordian species on the basis of the morphology of the developmental stages and spores as well as the pattern of organ, tissue, and cell involvement by the parasites. Nevertheless, electron microscopy will remain necessary for classification by genus or species.

Our findings suggest that the intestinal microsporidia $E$. bieneusi and $S$. intestinalis can be distinguished with use of coprodiagnostic techniques. In the patients reported here, we found microsporidian spores by light microscopic examination of stool specimens stained with chromotrope. The spores measuring 1.2 to $1.5 \mu \mathrm{m} \times 2.5$ to $3.0 \mu \mathrm{m}$ had the typical staining pattern described for microsporidia but appeared consistently and significantly bigger than those of $E$. bieneusi, which measure $\sim 0.9 \times 1.5 \mu \mathrm{m}$ [1]. Therefore, we performed an electron microscopic examination of stool specimens, which showed that the microsporidian spore ultrastructure differed from that of E. bieneusi (particularly in regard to the configuration of the coiled tubules) and was comparable with that described for Encephalitozoon species in urine specimens [7,8]. Since an Encephalitozoon species has never been identified in a human intestine and has not been associated with diarrhea in HIV-infected patients [7], we assumed that the patients had intestinal $S$. intestinalis infection; this was confirmed by electron microscopic examination of duodenal tissue for one of our patients [4]. The similarity of spores found in stool specimens and in duodenal

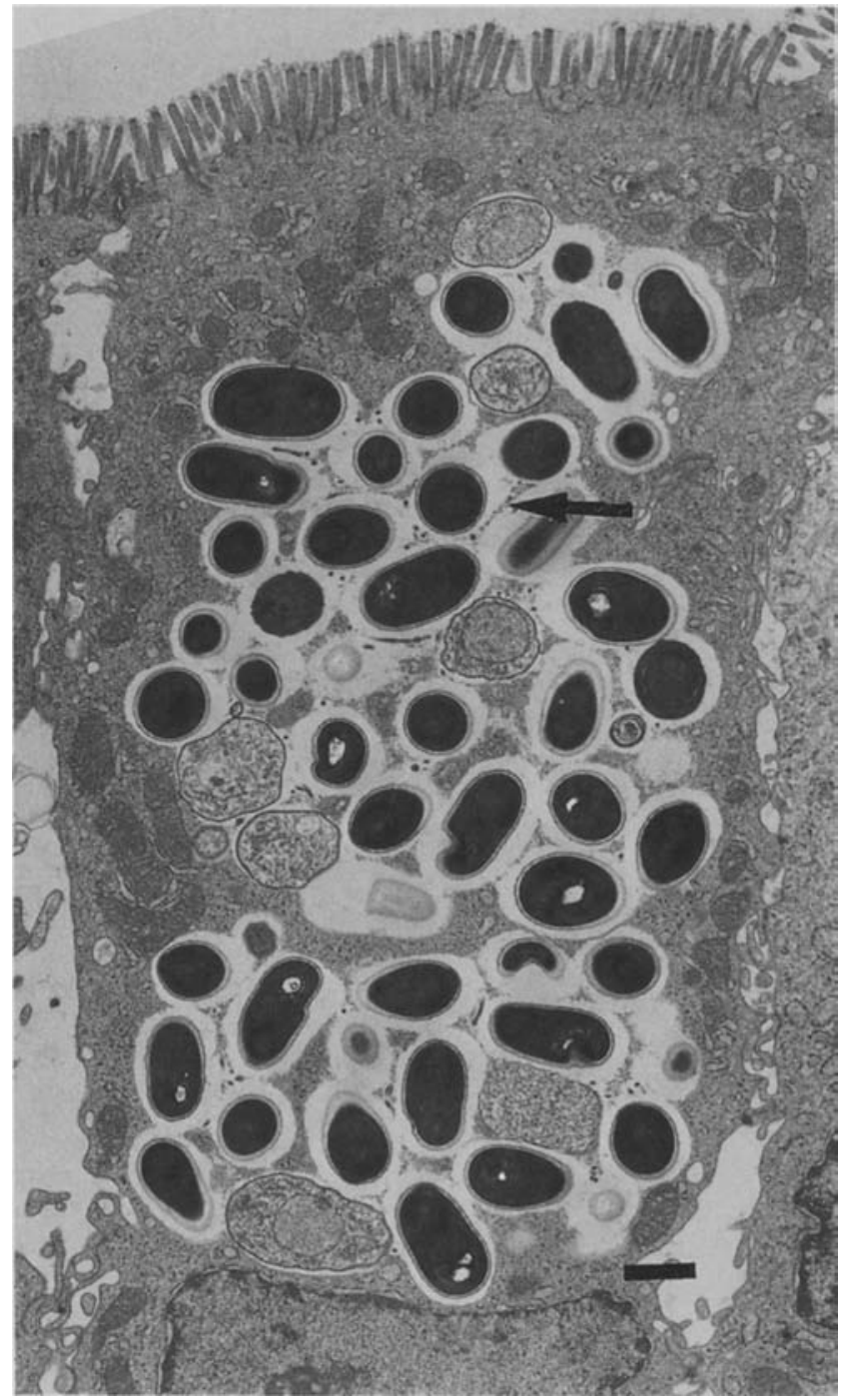

Figure 3. Electron micrograph of a duodenal tissue specimen showing clusters of sporonts and spores of $S$. intestinalis within a parasitophorous vacuole in an enterocyte. The parasitophorous vacuole appears to be septate by a fibrillar network (arrow) surrounding the developing organisms (bar $=1 \mu \mathrm{m}$ ).

tissue also was documented. In contrast with the observations of other investigators, who have found $S$. intestinalis to disseminate (particularly into the kidneys) in some patients [2-4], we found no clinical evidence of disseminated infection in our patients, and examinations of their urine samples were negative.

S. intestinalis has morphological and developmental characteristics comparable to those of Encephalitozoon species [2-4]. Indeed, spores of Encephalitozoon hellem identified in urine $[7,8,10]$ are very similar - perhaps identical—to those of $S$. intestinalis found in stool and urine specimens. The characteristics necessary to confirm that a microsporidian is unquestionably $S$. intestinalis have been described only in regard to tissue sections [2-4]. There are three distinctive 
features. (1) Both $S$. intestinalis and Encephalitozoon species develop intracellularly within a parasitophorous vacuole, but only $S$. intestinalis-infected cells show a specific parasitesecreted fibrillar network surrounding the developing organisms [4]. (2) Encephalitozoon species organisms are disporous; $S$. intestinalis is tetrasporous, i.e., elongated proliferative meronts are uninucleate, binucleate, or tetranucleate [4]. (3) In contrast with other microsporidia found in humans, E. bieneusi develops in direct contact with the enterocyte cell cytoplasm (no parasitophorous vacuole is present) and has a characteristic multinucleate merogonic stage.

Treatment of E. bieneusi infection thus far has not resulted in disappearance of parasites in intestinal tissue, cessation of spore excretion, or lasting clinical improvement [11]. In contrast, anecdotal observations of successful treatment of $S$. intestinalis infection have been reported [5]. In regard to our patients, we could demonstrate both clinical improvement and disappearance of parasites from stool specimens during a 2-week course of albendazole. Moreover, we have shown that light microscopic coprodiagnosis is very useful for monitoring the course of treatment as well as for follow-up.

Stool examination not only is a means for diagnosis of intestinal microsporidiosis but also may allow the identification of different microsporidian species. Specific treatment with albendazole of diarrhea associated with Septata species appears to be curative. Further studies have to determine whether maintenance therapy is necessary and what dosage would be appropriate.

\section{Acknowledgments}

The authors are indebted to Prof. Thomas Bächi and Dr. Werner Wunderli for technical support and to Sandra Opravil for editorial assistance.

\section{References}

1. Weber R, Bryan RT, Owen RL, Wilcox CM, Gorelkin L, Visvesvara GS. Improved light-microscopic detection of microsporidia spores in stool and duodenal aspirates. N Engl J Med 1992;326:161-6.

2. Orenstein JM, Tenner M, Cali A, Kotler IPP. A microsporidian previously undescribed in humans, infecting enterocytes and macrophages, and associated with diarrhea in an acquired immunodeficiency syndrome patient. Hum Pathol 1992;23:722-8.

3. Orenstein JM. Dieterich DT, Kotler DP. Systemic dissemination by a newly recognized intestinal microsporidia species in AIDS. AIDS 1992:6:1143-50.

4. Cali A, Kotler DP. Orenstein JM. Septata imestinalis N. G.. N. Sp. an intestinal microsporidian associated with chronic diarrhea and dissemination in AIDS patients. J Eukaryot Microbiol 1993;40: 101-12.

5. Orenstein JM, Dieterich DT, Kotler DP. Albendazole as a treatment for disseminated microsporidiosis due to Septata intestinalis in AIDS patients [abstract]. In: Program of the Workshop on Intestinal Microsporidia in HIV Infection. Paris: Unité INSERM 313.1992.

6. Weber R. Kuster H. Keller R, et al. Pulmonary and intestinal microsporidiosis in a patient with the acquired immunodeficiency syndrome. Am Rev Respir Dis 1992;146:1603-5.

7. Weber R. Kuster H, Visvesvara GS, Bryan RT, Schwartz DA, Lüthy R. Disseminated microsporidiosis due to Encephalitozon hellem: pulmonary colonization, microhematuria, and mild conjunctivitis in a patient with AIDS. Clin Infect Dis 1993;17:415-9.

8. Visvesvara GS, Leitch GJ, Moura H. Wallace S, Weber R, Bryan RT. Culture, electron microscopy, and immunoblot studies on a microsporidian parasite isolated from the urine of a patient with AIDS. J Protozool 1991;38:105S-11IS

9. Weber R. Sauer B, Lüthy R. Nadal D. Intestinal coinfection with Eimerocyoson bieneusi and criprosporidium in a human immunodeficiency virus-infected child with chronic diarrhea. Clin Infect Dis 1993:17:480-3

10. Didier ES. Didier PJ, Friedberg DN, et al. Isolation and characterization of a new human microsporidian. Encephalitoroon hellem (n. sp.). from three AIDS patients with keratoconjunctivitis. J Infect Dis 1991:163:617-21.

11. Blanshard C. Ellis DS. Tovey DG. Dowell S, Gazzard BG. Treatment of intestinal microsporidiosis with albendazole in patients with AIDS. AIDS 1992;6:311-3. 